\title{
New protection scheme for internal fault of multi-microgrid
}

\author{
Fan Zhang and Longhua $\mathrm{Mu}^{*}$
}

\begin{abstract}
Multi-microgrids have many new characteristics, such as bi-directional power flow, flexible operation and variable fault current consisting of the different control strategy of inverter interfaced distributed generations (IIDGs), which all present challenges in multi-microgrid protection. In this paper, the current and voltage characteristics of different feeders are analyzed considering faults at different locations of the multi-microgrid. Based on the voltage and current distribution characteristics of the line parameters, a new protection scheme for the internal faults of multi-microgrids is proposed, which takes the change of phase difference and amplitude of measured bus admittances as the criterion. This proposed scheme has high sensitivity and reliability, is based on a simple principle, and can be easily adjusted. Simulation results using PSCAD/EMTDC verify the correctness and effectiveness of the protection scheme.
\end{abstract}

Keywords: Microgrid, Multi-microgrid, Measured admittance, Protection scheme

\section{Introduction}

With increased energy demand and pressure to reduce emissions, microgrids have gradually become a hot research topic in power systems [1]. A microgrid is a medium or low-voltage power system, which consists of distributed generations (DGs), energy storage devices, energy conversion devices, loads, and corresponding supervision and protection equipment [2, 3]. Multi-microgrids are a new concept of distributed generation networks and connect microgrids that are geographically adjacent to each other [4-6] Developing multi-microgrids can help to implement energy complement in different operating conditions among various microgrids, and decrease difficulties in system scheduling. Furthermore, in certain control strategy and energy optimization management, multimicrogrids can improve the self-healing ability of power networks, ensuring continuous power supply to important consumers and increased network reliability. Thus, developing multi-microgrids can help promote better applications based on the present advantages of microgrids.

One of the key enabling technologies of the multimicrogrid is the protection scheme. The protection scheme of a traditional distribution network, including instantaneous trip current protection and overcurrent protection,

\footnotetext{
* Correspondence: Ihmu@tongji.edu.cn

Department of Electrical Engineering, Tongji University, Shanghai 201804, China
}

was designed based on distinct fault features such as the unidirectional power flow and the high level of the fault current. In this scheme, protection selectivity is achieved by time-delay cooperation. Unlike traditional distribution networks where there is only unidirectional power flow, there will be bi-directional power flow between the power grid and the multi-microgrid, and among the sub-microgrids. In addition, the DGs' currents are sometimes restricted by a fault current limiter [7, 8], whereas the fault level of multimicrogrids is usually very low $[9,10]$. Given all these factors, the protection scheme of traditional distribution networks cannot be simply transplanted into the multimicrogrid systems. Therefore, one major issue faced by researchers is how to design a protection system for multimicrogrids based on the real working condition.

At present, research on the protection of multi-microgrids is still at its early stage, however, some achievements have been made in the field. A protection scheme for island operated medium-voltage microgrids without the need for high-speed communication was proposed in [11]. The proposed scheme is based on the usage of an admittance related start value and is applicable to both short-circuit and earthfault protections, as well as to both inverter-based and directly connected synchronous generator based DG units. To solve the issue of conventional distribution network protections not being satisfactory to the requirements of microgrids without the aid of communication technology, an 
inverse-time low-impedance protection scheme based on load impedance was presented in [12]. Although this protection scheme can identify the faulted sections, it cannot locate the concrete branch feeders. A protection method utilizing the total admittance of the protected line segment was presented in [13]. Since the line length in active distribution networks is always short, the protection can only cover a very small part of the segment. An alternate protection and control scheme for microgrids with converter interfaced micro sources was proposed in [14], where a new inverse time relay characteristic was put forward by using the measured admittance of the protected line. The proposed protection and control scheme consider both grid connected and autonomous operation of the microgrid. However, the existence of harmonic and the transient behavior of the current introduce some problems on relay accuracy. Based on superimposed reactive energy and Hilbert transform, a protection scheme for microgrids was proposed in [15]. However, the fault resistance can present a considerable error on relay reading and accuracy. Meanwhile, the specific control strategy of DGs was not been considered.

In summary, the existing schemes cannot effectively solve multi-microgrids protection. There are two main problems: First, the control strategies of the DGs which have a large impact on the analysis of fault characteristics $[16,17]$, are ignored in most protection schemes. Second, the majority of the existing work focus on a single microgrid, with the interconnection and interaction among the adjacent microgrids not taken into consideration. For multi-microgrids, if each sub-microgrid is treated as a single DG, there is a bi-directional power exchange between itself and the outside zone. However, for a DG in a microgrid or active distribution network, the power exchange can only be from the DG to the outside zone, which is unidirectional. Therefore, the fault characteristics differ among the multi-microgrids, the active distribution networks, and the microgrids.

This paper proposes a new protection scheme for internal faults of multi-microgrids, which considers the control strategies of the DGs as well as the interconnection and interaction among the adjacent microgrids. A measured admittance scheme is proposed that analyzes the current and voltage vector graph before and after the fault. Fault characteristics of multi-microgrids can then be obtained and used to identify the faulty feeders. Finally, simulation results using PSCAD/EMTDC verify the correctness and effectiveness of the proposed protection scheme.

\section{Microgrid structure and a new algorithm for fault location}

Multi-microgrid is an emerging concept, and as shown in Fig. 1, each sub-microgrid is connected to the multi-microgrid at the point of common coupling (PCC). PCC is also the connection hub between the submicrogrid and the distribution network. According to the location of PCC, it can be divided into three types: the distribution network layer PCC (DNPCC), the multimicrogrid layer PCC (MPCC) and sub-microgrid layer PCC (SPCC). As shown in Fig. 1, DNPCC consists of DNPCC0-1, MPCC consists of MPCC1-4 and the SPCC consists of SPCC1-5. A multi-microgrid usually has two typical structures: radial structure and looped structure. If MPCC3 or MPCC4 is open, it is a radial structure, whereas when MPPC3 and MPCC4 are closed, it is a looped structure. Most of the existing multi-microgrid studies focus on the medium or lowvoltage radial structure $[18,19]$.

As shown in Fig. 1, multi-microgrids are connected to the distribution network through step-up transformers. In low-voltage side, there are several $\mathrm{AC}$ microgrids with different energy structures and load characteristics. The integration standard of a multi-microgrid follows the principles of $\mathrm{m}$ sub-microgrids interconnected together at below $35 \mathrm{kV}$ voltage level with the goal to achieve maximal flow optimization efficiency and minimal power failure probability. The multi-microgrid that consists of $\mathrm{m}$ sub-microgrids is connected to the distribution network with $\mathrm{n}$ interfaces. The sub-microgrids in the multimicrogrid are usually connected together in series and parallel, and are then accessed by the distribution network. This leads to the interface numbers usually being lower than the number of the sub-microgrids, i.e. $\mathrm{n}<\mathrm{m}$. As shown in Fig. 1, Sub-microgrids 1, 2 and 3 are in series connection, and Sub-microgrids 4 and 5 are also in series connection. The two series are then connected to the distribution network, and thus, in this example $n=2$ and $\mathrm{m}=5$.

When MPCC3 or MPCC4 is open, the multimicrogrid has similar structure to a traditional radial distribution system, though some differences exist. In the multi-microgrid, each sub-microgrid can be regarded as a protection zone, in which there exists bidirectional power exchange between the submicrogrid and the outside zone. However, for the traditional radial distribution system, the power exchange is unidirectional from the distribution system to the outside zone. The connection and disconnection of sub-microgrids in the multi-microgrid, and the connection and disconnection of DGs or controllable loads in each of the sub-microgrids will change topology of the multi-microgrid leading to variable operation modes. Therefore, the fault characteristics differ between the multi-microgrid and traditional radial distribution system.

By controlling the open and close of the PCC, the coordinated control and protection of the whole multi- 


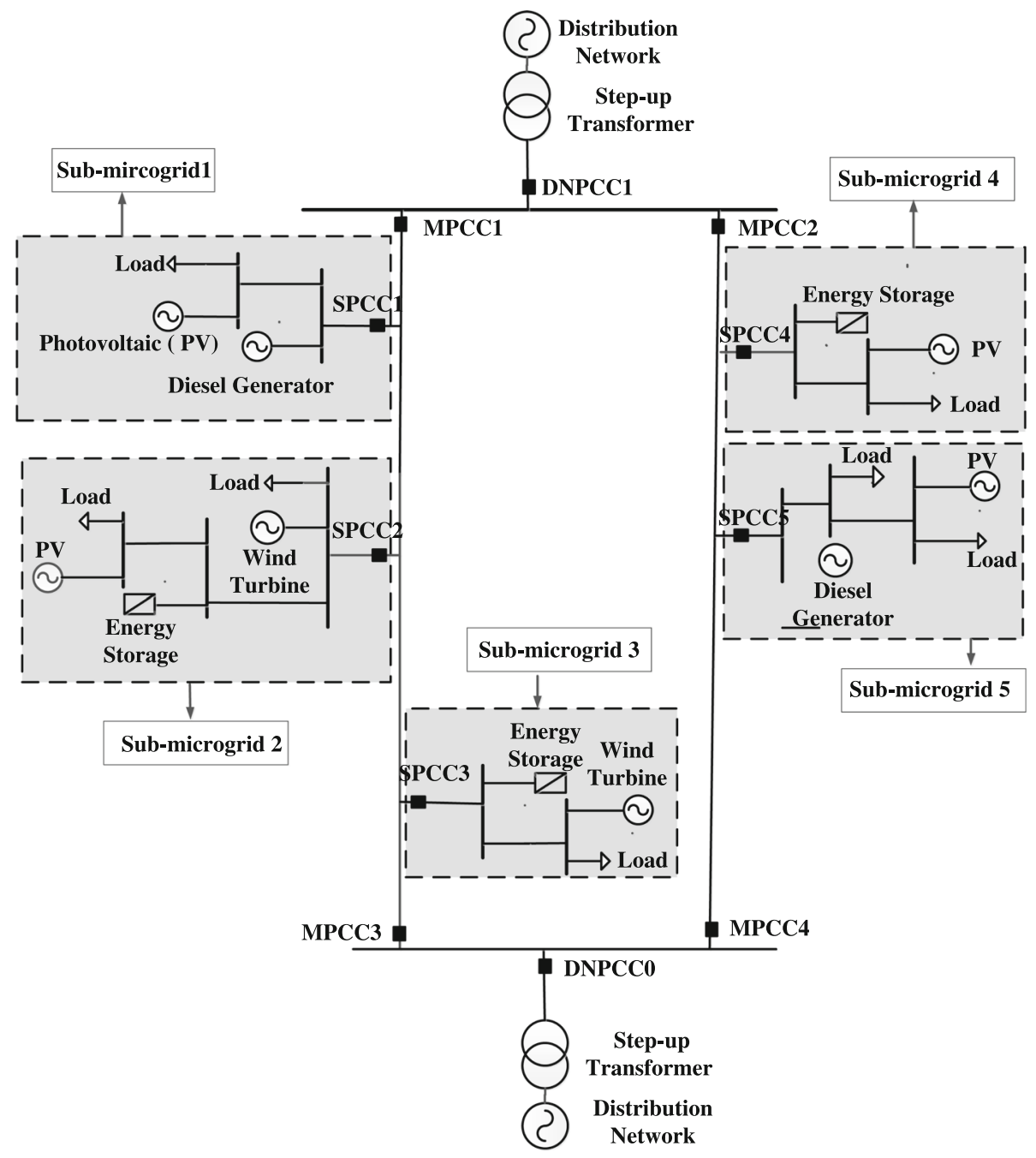

Fig. 1 Schematic diagram of the multi-microgrid system

microgrid can be achieved. The multi-microgrid has the following three operation modes.

(1) When there is no fault, it operates in grid-connected mode.

(2) If a fault occurs in the distribution network, the multi-microgrid changes into islanded mode.

(3) For the internal fault of the multi-microgrid, the faulty sub-microgrid will be disconnected and the healthy ones remain operational in grid-connected mode.

A simplified diagram of Fig. 1 is shown as Fig. 2 when MPCC3-4 are open. For grid-connected operation, SPCC1 to SPCC5 are closed. This paper mainly discusses the protection scheme for internal faults of the multi-microgrid with radial structure in grid-connected mode, where DGs are under PQ control strategy. The feeders in the multi-microgrid are divided into the following two categories.

(1) The double-terminal feeder: the tie lines between the sub-microgrids (such as feeders $A B, B C, C D$, $\mathrm{EF}, \mathrm{FG}$ ), and the tie lines between two buses (such as the feeders $\mathrm{HM}, \mathrm{NQ}$, etc.);

(2) The single-terminal feeder: the feeders connecting to DGs (called DG feeders in this paper) including feeders L1, L3, and etc., and the feeders connecting to loads (called load feeders) including feeders L2, L4, and etc.

As seen in Fig. 2, when faults occur in the doubleterminal feeder BC and single-terminal feeders L3 and L2, the change of measured bus admittances before and after the fault occurrence are analyzed, and are used to identify fault feeder selection criterion. 


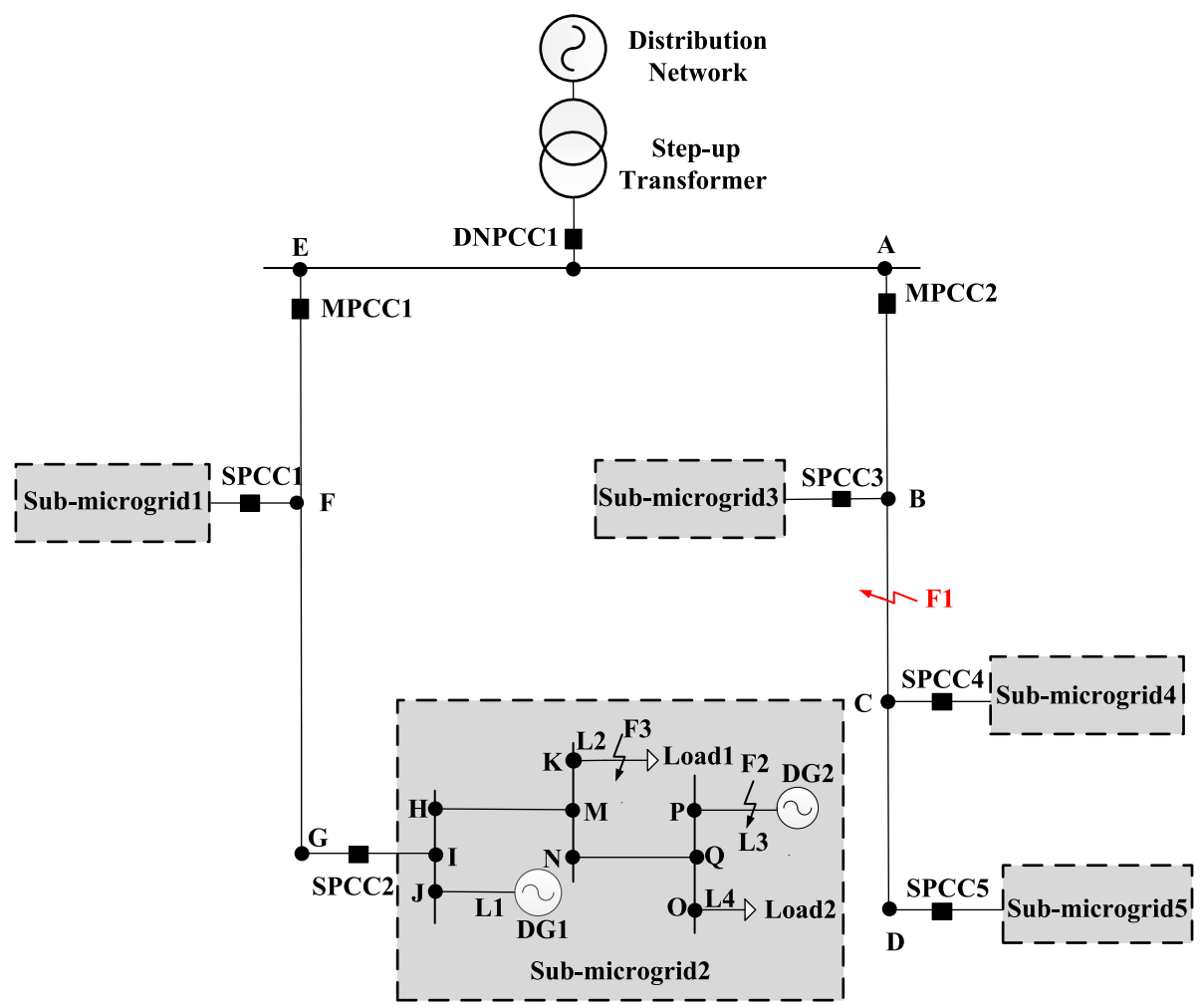

Fig. 2 Simplified structure of multi-microgrid system

For analysis, a simplified line diagram is shown in Fig. 3. Set the forward direction of current from bus to feeder, and denote $\dot{U}_{\mathrm{X}}, \dot{I}_{\mathrm{X}}, G_{\mathrm{X}}$ and $\dot{U}_{1 \mathrm{X}}, \dot{I}_{1 \mathrm{X}}, G_{1 \mathrm{X}}$ as the voltages, currents and measured admittances of $X$ terminal before and after the fault occurrence, respectively, the power flow change of $X$ terminal can be defined into four situations.

The phase and phase difference of pre- and post-fault measured admittances of $X$ terminal are:

$$
\begin{aligned}
& \Delta \phi_{1}=\arg \left(G_{X}\right)=\arg \left(\dot{I}_{X}\right)-\arg \left(\dot{U}_{X}\right) \\
& \Delta \phi_{2}=\arg \left(G_{1 X}\right)=\arg \left(\dot{I}_{1 X}\right)-\arg \left(\dot{U}_{1 X}\right) \\
& \Delta \phi_{X}=\Delta \phi_{2}-\Delta \phi_{1}
\end{aligned}
$$

where $\arg (\cdot)$ is the symbol of phase angle. $\Delta \phi_{X}$ is the phase difference of the pre- and post-fault measured

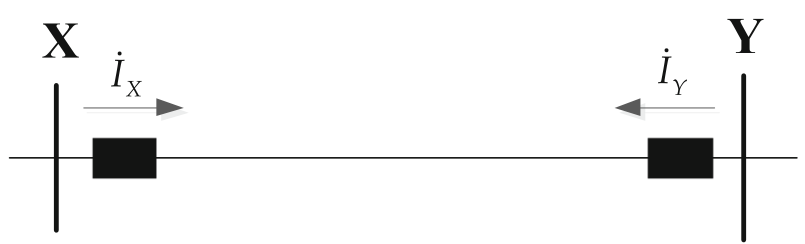

Fig. 3 Current direction of a simplified line admittances of $X$ terminal. All the phase is converted to the range from $-90^{\circ}$ to $270^{\circ}$.

Situation (1): Both the pre- and post-fault current of $X$ terminal are in forward direction and do not change. The current and voltage vector graphs are shown in Fig. 4.

It is seen from Fig. 4 that:

$$
\left\{\begin{array}{l}
-90^{\circ} \leq \Delta \phi_{1} \leq 0^{\circ} \\
-90^{\circ} \leq \Delta \phi_{2} \leq 0^{\circ}
\end{array}\right.
$$

Thus,

$$
-90^{\circ} \leq \Delta \phi_{X} \leq 90^{\circ}
$$

Situation (2): Both the pre- and post-fault current of $X$ terminal are in reverse direction and do not change. The current and voltage vector graph is shown in Fig. 5.

It is known from Fig. 5 that
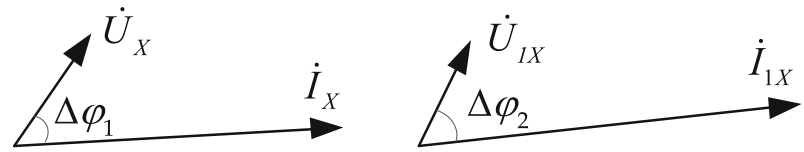

Fig. 4 The pre- and post-fault current and voltage vector graphs of $X$ terminal of situation (1) 


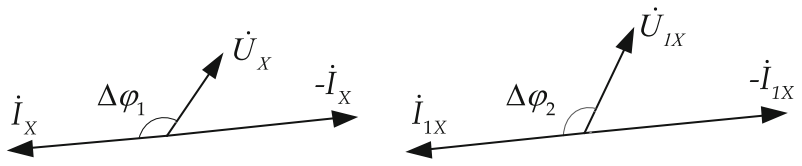

Fig. 5 The pre- and post-fault current and voltage vector graphs of $X$ terminal of situation (2)

$$
\left\{\begin{array}{l}
90^{\circ} \leq \Delta \phi_{1} \leq 180^{\circ} \\
90^{\circ} \leq \Delta \phi_{2} \leq 180^{\circ}
\end{array}\right.
$$

Thus,

$$
-90^{\circ} \leq \Delta \phi_{X} \leq 90^{\circ}
$$

Situation (3): The pre-fault current direction of $X$ terminal is forward, but becomes reverse direction after the fault occurrence. The current and voltage vector graphs are shown in Fig. 6.

It is known from Fig. 6 that:

$$
\left\{\begin{array}{c}
-90^{\circ} \leq \Delta \phi_{1} \leq 0^{\circ} \\
90^{\circ} \leq \Delta \phi_{2} \leq 180^{\circ}
\end{array}\right.
$$

Thus,

$$
90^{\circ} \leq \Delta \phi_{X} \leq 270^{\circ}
$$

Situation (4): The per-fault current of $X$ terminal is in reverse direction, but becomes forward direction after the fault occurrence. The current and voltage vector graph is shown in Fig. 7. It is known from Fig. 7 that:

$$
\left\{\begin{array}{c}
90^{\circ} \leq \Delta \phi_{1} \leq 180^{\circ} \\
-90^{\circ} \leq \Delta \phi_{2} \leq 0^{\circ}
\end{array}\right.
$$

Thus,

$$
-270^{\circ} \leq \Delta \phi_{X} \leq-90^{\circ}
$$

According to the phase conversion principle, the phase difference in (11) changes to:

$$
90^{\circ} \leq \Delta \phi_{X} \leq 270^{\circ}
$$

Based on the above analysis, the phase difference change information of the pre- and post-fault measured admittances of $X$ terminal is shown in Table 1 .
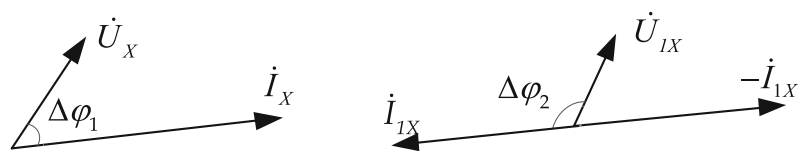

Fig. 6 The pre- and post-fault current and voltage vector graphs of $X$ terminal of situation (3)

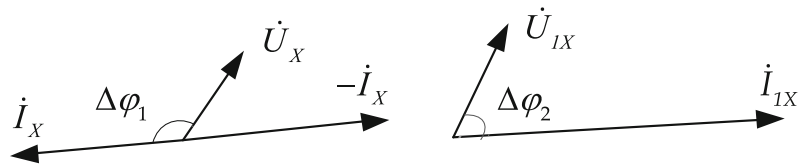

Fig. 7 The pre- and post-fault current and voltage vector graphs of $X$ terminal of situation (4)

\section{Fault analysis for multi-microgrid with radial structure}

3.1 Fault at double-terminal feeder

3.1.1 Fault analysis for the fault double-terminal feeder

The analysis for fault occurring at the double-terminal feeder uses the $\mathrm{F} 1$ fault in line $\mathrm{BC}$ as an example. In normal operation, the power flow of feeder $\mathrm{BC}$ consists of the following two situations.

(a) The load demand of Sub-microgrids 4 and 5 is greater than the generation capacity of DGs, and thus the power flow is from $B$ terminal to $C$ terminal.

(b) The load demand of Sub-microgrids 4 and 5 is less than the generation capacity of DGs, and thus the power flow is from $C$ terminal to $B$ terminal.

\section{Situation}

(a): $\dot{U}_{B}, \dot{I}_{B}$ and $\dot{U}_{1 B}, \dot{I}_{1 B}$ are the pre- and post-fault voltages and currents of $B$ terminal in line $B C$, respectively. For $B$ terminal, it is in the upstream of the fault point and is connected with the distribution network, so the voltage will largely remain unchanged when the fault occurs but the current will significantly increase. The variation of pre- and post-fault current and voltage are:

$$
\left|\dot{U}_{1 B}\right| \leq\left|\dot{U}_{B}\right|
$$

$$
\left|\dot{I}_{B}\right| \leq\left|\dot{I}_{1 B}\right|
$$

The pre- and post-fault measured admittances of $B$ terminal are:

$$
G_{B}=\dot{I}_{B} / \dot{U}_{B}
$$

$$
G_{1 B}=\dot{I}_{1 B} / \dot{U}_{1 B}
$$

Table 1 Phase difference change information of measured admittances of $X$ terminal before and after the fault

\begin{tabular}{lllll}
\hline Direction & Forward- & Reverse- & Forward- & Reverse- \\
Measured & Forward & Reverse & Reverse & Forward \\
Admittance & Situation (1) & Situation (2) & Situation (3) & Situation (4) \\
\hline Phase Difference & $-90^{\circ}-90^{\circ}$ & $-90^{\circ}-90^{\circ}$ & $90^{\circ}-270^{\circ}$ & $90^{\circ}-270^{\circ}$ \\
\hline
\end{tabular}


Combining (13)-(16), it is known that the measured admittance amplitudes have the following relationship:

$$
\left|G_{1 B}\right|>>\left|G_{B}\right|
$$

Both the pre- and post-fault current directions of $B$ terminal are in the forward direction. It is also known from Table 1 that the phase difference of measured admittances of $B$ terminal is:

$$
-90^{\circ} \leq \Delta \phi_{B} \leq 90^{\circ}
$$

After the fault occurrence, the DGs in the downstream of $C$ terminal cannot provide enough short circuit capacity, so the voltage of $C$ terminal will drop significantly. Additionally, the DGs in the sub-microgrids are inverter interfaced distributed generators (IIDG), which are usually under PQ control strategy with low voltage ride-through capability. When the voltage of the fault point drops significantly, the output current of the IIDG may reverse and decrease in amplitude [20]. Thus, the traditional current protection scheme is probably invalid in this case. In addition, if the DG penetration reaches a certain level, the protection cooperation will also be interfered. Meanwhile, the amplitude change of the measured pre- and post- fault admittances of $C$ terminal cannot be estimated directly with (15) and (16).

The pre-fault current of $C$ terminal is in reverse direction, and when the fault occurs, it changes to forward direction. Thus, the phase difference of measured admittances of $C$ terminal is:

$$
90^{\circ} \leq \Delta \phi_{C} \leq 270^{\circ}
$$

Situation (b): In this case, the change information of measured admittance amplitude of $B$ terminal is the same as (17). Before the fault, the current of $B$ terminal is in reverse direction and when the fault occurs, it changes to forward direction. Thus, the phase difference of measured admittances of $B$ terminal is:

$$
-90^{\circ} \leq \Delta \phi_{B} \leq 90^{\circ}
$$

The pre- and post-fault current of $C$ terminal are both in forward direction. Thus, the phase difference of measured admittances of $C$ terminal is:

$-90^{\circ} \leq \Delta \phi_{C} \leq 90^{\circ}$

\subsubsection{Fault analysis for the healthy double-terminal feeder}

For fault occurrences in F1, the analysis for healthy double-terminal feeders uses line $\mathrm{AB}$ as an example. Before the fault, the power flow of line $A B$ consists of the following two situations.

(a) The load demand of Sub-microgrids 3, 4 and 5 is greater than the generation capacity of DGs, and thus the power flow is from $A$ terminal to $B$ terminal.

(b) The load demand of Sub-microgrid 3, 4 and 5 is less than the generation capacity of DGs, and thus the power flow is from $B$ terminal to $A$ terminal.

Situation (a): $G_{\mathrm{A}}, G_{1 \mathrm{~A}}$ and $G_{\mathrm{B} 1}, G_{1 \mathrm{~B} 1}$ are the pre- and post-fault measured admittances of $A$ and $B$ terminals, respectively. In this case, $A$ and $B$ terminals are in the upstream of the fault point, and are connect to the distribution network. Therefore, the voltage will remain unchanged when the fault occurs, but the current will increase significantly. Thus, there are:

$$
\begin{gathered}
\left|G_{1 A}\right|>>\left|G_{A}\right| \\
\left|G_{1 B 1}\right|>>\left|G_{B 1}\right|
\end{gathered}
$$

Both the pre- and post-fault current of $A$ terminal are in forward direction, while for $B$ terminal, they are both in reverse direction. Thus, the respective phase differences of measured admittances of $A$ and $B$ terminal are:

$$
\begin{aligned}
& -90^{\circ} \leq \Delta \phi_{A} \leq 90^{\circ} \\
& -90^{\circ} \leq \Delta \phi_{B} \leq 90^{\circ}
\end{aligned}
$$

Situation (b): $A$ and $B$ terminals are in the upstream of the fault point, so there are:

$$
\begin{gathered}
\left|G_{1 A}\right|>>\left|G_{A}\right| \\
\left|G_{1 B 1}\right|>>\left|G_{B 1}\right|
\end{gathered}
$$

The pre-fault current of $A$ terminal is in reverse direction, whereas it is in forward direction for $B$ terminal. When the fault occurs, the current of $A$ terminal changes to forward direction and $B$ terminal becomes reverse direction. Thus, the respective phase differences of measured admittance of $A$ and $B$ terminal are:

$$
\begin{aligned}
& 90^{\circ} \leq \Delta \phi_{A} \leq 270^{\circ} \\
& 90^{\circ} \leq \Delta \phi_{B} \leq 270^{\circ}
\end{aligned}
$$

The power flow analysis for other healthy doubleterminal feeders is similar to feeder $A B$, and thus is not covered here.

\subsubsection{Fault analysis for single-terminal feeder}

In this case, the current and voltage remain unchanged, and thus the measured admittance amplitudes will not change after the fault occurrence.

For the DG feeders, the current directions are from the DGs to the upstream bus, and all are in the reverse direction. Thus, the phase difference of measured admittances is:

$$
-90^{\circ} \leq \Delta \phi_{D G} \leq 90^{\circ}
$$

For the load feeders, their current directions are from the upstream buses to the loads, and are all in 
forward direction. So the phase difference of measured admittances is:

$$
-90^{\circ} \leq \Delta \phi_{\text {Load }} \leq 90^{\circ}
$$

\subsection{Fault occurs in single-terminal feeder}

The analysis for fault occurrence in a single-terminal feeder uses the F2-fault of feeder L3 and F3-fault of feeder L2 as examples.

\subsubsection{Fault at F2}

For faults occurring in F2, (Fig. 2), DG2 is connected to feeder L3, and $P$ terminal is the access point that L3 connects with the upstream bus. The pre-fault current direction of $P$ terminal is from DG2 to the upstream bus, and is in reverse direction. After the fault occurrence, it changes to forward direction from the upstream bus to the fault point. Thus, the phase difference of measured admittances is:

$$
90^{\circ} \leq \Delta \phi_{P} \leq 270^{\circ}
$$

The power flow analysis for the other healthy feeders is the same as the feeder in Section 3.1 so not repeated here.

\subsubsection{Fault at F3}

For fault at F3 (Fig. 2), load2 is connected to feeder L2, and $K$ terminal is the access point that $\mathrm{L} 2$ connects with the upstream bus. The pre- and post-fault current directions of $K$ terminal are all in forward direction from the upstream bus to the fault point. Thus, the phase difference of measured admittances is:

$$
-90^{\circ} \leq \Delta \phi_{K} \leq 90^{\circ}
$$

$K$ terminal is in the upstream of the fault point, so the post-fault measured admittance amplitude of $K$ terminal will increase significantly. For the other feeders that connect with the loads, the current and voltage will remain unchanged, and thus the post-fault measured admittance amplitude will not change.

The power flow analysis for the other feeders is similar to the feeders in Section 3.1, so not repeated here.

\section{Protection scheme for internal fault of multi- microgrid}

\subsection{Criterion for fault location}

By analyzing the change of the measured admittances before and after the fault occurrence, the following results are obtained.

(1) Fault occurs in double-terminal feeder
The phase differences of measured admittances of the two terminals are in the ranges of $90^{\circ}-270^{\circ}$ and $90^{\circ}-90^{\circ}$, respectively. For the healthy double-terminal feeders, the phase differences of the two terminals are both in the range of either $90^{\circ}-270^{\circ}$ or $-90^{\circ}-90^{\circ}$. For the single-terminal feeders, the phase difference range is in $-90^{\circ}-90^{\circ}$.

\section{(2) Fault occurs in single-terminal feeder}

If the fault occurs in the DG feeders, the phase difference of measured admittances is in the range of $90^{\circ}-270^{\circ}$. For the double-terminal feeders, the phase difference is in the range of either $90^{\circ}-270^{\circ}$ or $-90^{\circ}-90^{\circ}$. For the other single-terminal feeders, the phase difference range is $-90^{\circ}-90^{\circ}$.

If the fault occurs in the load feeders, the phase difference of double-terminal feeders is in the range of either $90^{\circ}-270^{\circ}$ or $-90^{\circ}-90^{\circ}$. For the single-terminal feeders, the phase difference range is $-90^{\circ}-90^{\circ}$.

From the above analysis, a solution can be easily obtained to identify the faulty feeder only by the change of the phase difference of measured admittances for faults in either the DG feeders or doubleterminal feeders.

However, for faults in the load feeders, there is no obvious fault feature for the change of the phase difference of measured admittances, as they are in the same range before and after the fault. Based on the above analysis, the load feeders only have connected loads and their power is provided only by the upstream buses. When the fault occurs, there will be large increase in current but significant drop in voltage. Thus, the measured admittance amplitude will increase significantly and can be selected as the fault criterion for the load feeders.

It can be seen in Table 2 that regardless of the fault location, the measured admittance amplitude of the fault feeder always increases. Thus, the measured admittance amplitude is selected as the starting criterion as:

$$
G_{\mathrm{i}}>2 G_{\mathrm{R}}
$$

where $G_{\mathrm{R}}$ and $G_{\mathrm{i}}$ are the measured admittance amplitudes of feeders before and after fault occurrence, respectively.

Overall, the following fault criteria are used to detect the internal fault of multi-microgrids.

Criterion 1: DG Feeders. The phase difference $\Delta \phi_{1}$ of measured admittances is:

$$
90^{\circ} \leq \Delta \phi_{1} \leq 270^{\circ}
$$


Table 2 Change information of measured admittance

\begin{tabular}{|c|c|c|c|c|c|c|c|c|}
\hline \multirow{2}{*}{\multicolumn{3}{|c|}{$\frac{\text { Fault Position }}{\text { Measured Admittance }}$}} & \multicolumn{2}{|l|}{$\mathrm{F} 1$} & \multicolumn{2}{|l|}{ F2 } & \multicolumn{2}{|l|}{ F3 } \\
\hline & & & \multirow{2}{*}{$\begin{array}{l}\Delta \varphi \\
90^{\circ}-270^{\circ} \\
\text { and }-90^{\circ}-90^{\circ}\end{array}$} & \multirow{2}{*}{$\begin{array}{l}\frac{\left|G_{i}\right|}{\text { Increases }} \\
\text { Significantly }\end{array}$} & \multirow{2}{*}{$\begin{array}{l}\Delta \varphi \\
-\end{array}$} & \multirow{2}{*}{$\frac{\left|G_{i}\right|}{-}$} & \multirow{2}{*}{$\begin{array}{l}\Delta \varphi \\
-\end{array}$} & \multirow{2}{*}{$\frac{\left|G_{i}\right|}{-}$} \\
\hline $\begin{array}{l}\text { Double-terminal } \\
\text { Feeder }\end{array}$ & & Fault & & & & & & \\
\hline & & Healthy & $\begin{array}{l}90^{\circ}-270^{\circ} \\
\text { or }-90^{\circ}-90^{\circ}\end{array}$ & $\begin{array}{l}\text { Basically } \\
\text { Unchanged }\end{array}$ & $\begin{array}{l}90^{\circ}-270^{\circ} \\
\text { or }-90^{\circ}-90^{\circ}\end{array}$ & $\begin{array}{l}\text { Basically } \\
\text { Unchanged }\end{array}$ & $\begin{array}{l}90^{\circ}-270^{\circ} \\
\text { or }-90^{\circ}-90^{\circ}\end{array}$ & $\begin{array}{l}\text { Basically } \\
\text { Unchanged }\end{array}$ \\
\hline \multirow[t]{4}{*}{$\begin{array}{l}\text { Single- terminal } \\
\text { Feeder }\end{array}$} & DG Feeder & Fault & - & - & $90^{\circ}-270^{\circ}$ & $\begin{array}{l}\text { Increases } \\
\text { Significantly }\end{array}$ & - & - \\
\hline & & Healthy & $-90^{\circ}-90^{\circ}$ & $\begin{array}{l}\text { Basically } \\
\text { Unchanged }\end{array}$ & $-90^{\circ}-90^{\circ}$ & $\begin{array}{l}\text { Basically } \\
\text { Unchanged }\end{array}$ & $-90^{\circ}-90^{\circ}$ & $\begin{array}{l}\text { Basically } \\
\text { Unchanged }\end{array}$ \\
\hline & Load Feeder & Fault & - & - & $-90^{\circ}-90^{\circ}$ & $\begin{array}{l}\text { Basically } \\
\text { Unchanged }\end{array}$ & $-90^{\circ}-90^{\circ}$ & $\begin{array}{l}\text { Increases } \\
\text { Significantly }\end{array}$ \\
\hline & & Healthy & $-90^{\circ}-90^{\circ}$ & $\begin{array}{l}\text { Basically } \\
\text { Unchanged }\end{array}$ & - & - & $-90^{\circ}-90^{\circ}$ & $\begin{array}{l}\text { Basically } \\
\text { Unchanged }\end{array}$ \\
\hline
\end{tabular}

Criterion 2: Double-terminal feeder. The phase differences $\Delta \phi_{2}$ and $\Delta \phi_{3}$ of measured admittances are:

$$
\begin{aligned}
& -90^{\circ} \leq \Delta \phi_{2} \leq 90^{\circ} \\
& 90^{\circ} \leq \Delta \phi_{3} \leq 270^{\circ}
\end{aligned}
$$

Criterion 3: Load feeders. The measured admittance amplitude of load feeders increase significantly after the fault, and exceeds a particular threshold $G_{\text {set }}$, which is 4-5 times bigger than that before fault occurrence.

\subsection{The protection scheme of multi-microgrid}

The flow chart of protection scheme is shown in Fig. 8 and the basic process is described as follows:

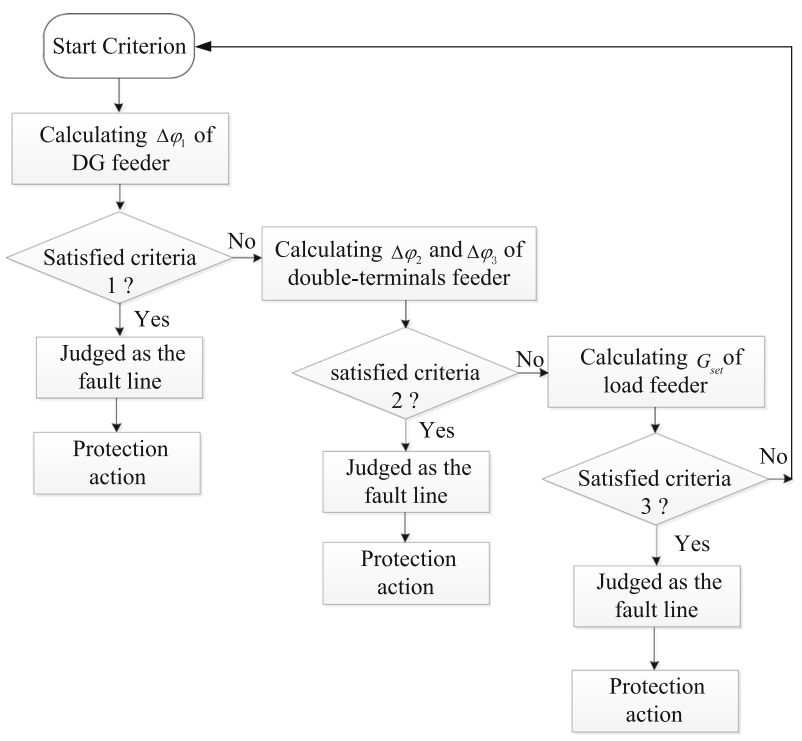

Fig. 8 Flow chart of the protection scheme
(1) When the start criterion is satisfied, the phase difference of measured admittances $\Delta \phi_{1}$ of the DG feeders is calculated. When Criteria 1 is met, the DG feeder is judged as the fault line.

(2) If Criteria 1 is not satisfied, the phase differences of measured admittances $\Delta \phi_{2}$ and $\Delta \phi_{3}$ of doubleterminal feeders are calculated. When Criteria 2 is met, the feeder is judged as the fault line.

(3) If Criteria 2 is not met, the measured admittance amplitude of load feeders is calculated. When Criteria 3 is met, the load feeder is judged as the fault line.

(4) Protection activates and the fault feeder is removed.

The measured information only contains the values of voltage and current before and after fault occurrence. So the disposal and transmission burden of data is low and the communication requirement is reduced. Besides, the lines in multi-microgrids are usually very short, so realtime data transfer can be achieved relatively easily. Therefore, the protection speed can be satisfied. At present, many signal acquisition and protection units have communication interfaces and thus, it is convenient to integrate these devices into the protection system.

Meanwhile, the measured admittance amplitude of the feeders satisfying the starting criterion $G_{i}$ is determined as the suspected faulty feeders. Then, the fault criterion of suspected faulty feeders is calculated. Only the feeder which satisfies the fault criterion is determined as the faulty line. The protection scheme only needs upload the information of the suspected feeders, and the traffic for decision-making is therefore reduced.

\subsection{Applicability analysis for the looped structure multi- microgrid}

When MPCC3 and MPCC4 are closed, the multimicrogrid is of looped structure. The double-terminal 
feeder $\mathrm{BC}$ will be taken for fault analysis as an example. Before fault occurrence, the power flows of B terminal and $\mathrm{C}$ terminal are both from either the upstream terminal of the fault point to the downstream terminal or from the downstream terminal to the upstream terminal. After fault occurrence, the power flow of B terminal is from upstream terminal to the fault point, whereas the power flow of $\mathrm{C}$ terminal is from downstream terminal to the fault point. According to the phase difference change information of measured admittances in Table 1, the following conclusions can be drawn: the phase differences of measured admittances of the $B$ and $C$ terminals are in the ranges of $90^{\circ}-270^{\circ}$ and $-90^{\circ}-90^{\circ}$, respectively. Thus, the fault analysis for the phase difference change information of looped structure multi-microgrid is the same as that of radial structure multi-microgrid.

In this situation, both $\mathrm{B}$ and $\mathrm{C}$ terminals are connected to the distribution network, so the voltages of the two terminals will have small changes when the fault occurs, but the currents will increase significantly. Thus, the measured admittance amplitudes of the two terminals will see large increases. While for the radial structure multi-microgrid, the DGs in the downstream of $\mathrm{C}$ terminal cannot provide enough short circuit capacity. As has been analyzed in the paper, the admittance amplitude change of $\mathrm{C}$ terminal cannot be estimated directly. So the phase amplitude change information of looped structure multi-microgrid will differ slightly from that of the radial structure multi-microgrid. The measured admittance amplitude information can be chosen as the starting criterion as the value for the faulty feeder always increases. Thus, although the range of fault starting detection has increased slightly, the faulty line can be isolated properly.

Overall, the proposed protection scheme can be applied to multi-microgrids with either radial structure or looped structure.

\section{Simulation results}

To validate the effectiveness and feasibility of the proposed method, a multi-microgrid with a high amount of DGs shown in Fig. 9 is established in PSCAD/EMTDC.

The multi-microgrid consists of three $10 \mathrm{kV}$ submicrogrids, and is connected to the main $35 \mathrm{kV}$ distribution network through a step-up transformer. The capacity and frequency of the system are $100 \mathrm{MVA}$ and $50 \mathrm{~Hz}$, respectively. The multi-microgrid has a high amount of DGs and loads, while for the purpose of analysis, the adjacent DGs and loads are converted to one high-capacity DG and load, respectively. Thus, each sub-microgrid only consists of one DG in Fig. 9. The DGs in each submicrogrid are the IIDGs under PQ control strategy with low voltage ride-through capability. The capacities of the DGs and loads in each sub-microgrid are shown in Table 3. The positive-sequence resistance and reactance of the

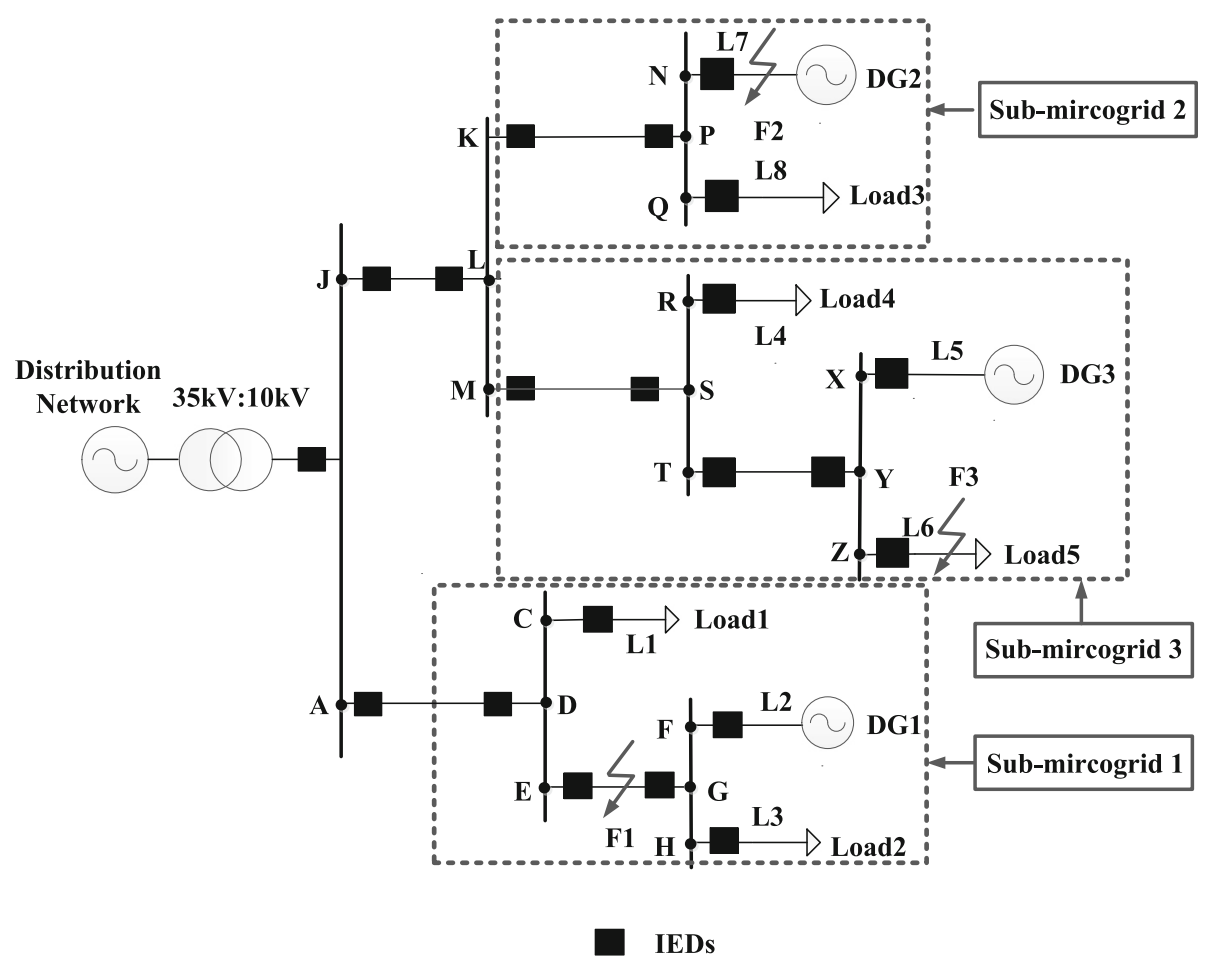

Fig. 9 Simulation model of the multi-microgrid 
Table 3 Capacity of DGs and loads in the multi-microgrid

\begin{tabular}{lll}
\hline $\begin{array}{l}\text { Number of } \\
\text { Multi-Microgrid }\end{array}$ & Loads & DGs \\
\hline 1 & LD1: $(1500+15 j) k V A$ & DG1: $500 \mathrm{~kW}$ \\
& LD2: $(1200+30 \mathrm{j}) \mathrm{kVA}$ & \\
3 & LD3: $(1200+30 \mathrm{j}) \mathrm{kVA}$ & DG2: $400 \mathrm{~kW}$ \\
& LD4: $(1200+30 \mathrm{j}) \mathrm{kVA}$ & DG3: $600 \mathrm{~kW}$ \\
& LD5: $(900+27 \mathrm{j}) \mathrm{kVA}$ & \\
\hline
\end{tabular}

feeders are $0.38 \Omega / \mathrm{km}$ and $0.45 \Omega / \mathrm{km}$, respectively, and the zero-sequence resistance and reactance are $0.76 \Omega / \mathrm{km}$ and $1.32 \Omega / \mathrm{km}$, respectively. Intelligent electronic devices (IEDs) are installed at the beginning of the single-terminal feeders. For double-terminal feeders, IEDs are installed at the two terminals of each line.

Three fault points (namely F1, F2 and F3) are selected to show faults in different sections of the multi-microgrid, i.e., the F1 fault at the double-terminal feeder EG, the F2 fault at the DG feeder L7 connecting with DG2, and the F3 fault in load feeder L6 connecting with load 5. Phasephase-to-ground fault is considered and the faults occur at $0.3 \mathrm{~s}$ after the simulation started. The transition resistance of the fault point is $2 \Omega$. The amplitude and phase difference change information of measured admittances before and after the fault are analyzed.

\subsection{Fault at F1}

The pre-fault measured admittances of the multimicrogrid are shown in Table 4. When the F1 fault occurs, the measured admittances of the multi-microgrid are shown in Table 5. $\left|G_{\mathrm{i}}\right|$ and $\Delta \phi_{1 \mathrm{i}},\left|G_{1 \mathrm{i}}\right|$ are the preand post-fault phase and amplitude of measured admittances, respectively. $\Delta \phi$ is the phase difference of the measured admittances.

In this case, it can be seen from Tables 4 and 5 that the pre- and post-fault phase differences of the measured admittances of $E$ and $G$ terminals are $-48.64^{\circ}$ and $150.99^{\circ}$, respectively, and they satisfy Criterion 2 . For the

Table 4 Pre-fault measured admittance results before fault

\begin{tabular}{|c|c|c|c|c|c|c|c|}
\hline Feeder & Terminal & $\left|G_{i}\right|$ & $\Delta \varphi_{\mathrm{i}}(\mathrm{O})$ & Feeder & Terminal & $\left|G_{i}\right|$ & $\overline{\Delta \varphi_{i}(\mathrm{O})}$ \\
\hline \multirow[t]{2}{*}{$\overline{\mathrm{EG}}$} & $E$ & 0.004 & 7.14 & L5 & $x$ & 0.003 & 172.92 \\
\hline & G & 0.004 & 186.89 & L6 & Z & 0.009 & -1.76 \\
\hline L1 & $C$ & 0.015 & -1.13 & L7 & $N$ & 0.006 & 175.1 \\
\hline L2 & $F$ & 0.015 & 173.82 & TY & $T$ & 0.006 & -1.93 \\
\hline L3 & $H$ & 0.012 & -1.49 & & Y & 0.006 & 176.92 \\
\hline L4 & $R$ & 0.012 & -1.76 & L8 & $Q$ & 0.012 & -1.66 \\
\hline \multirow[t]{2}{*}{$\mathrm{KP}$} & K & 0.006 & 170.21 & $A D$ & $A$ & 0.019 & -0.4 \\
\hline & P & 0.006 & -2.03 & & $D$ & 0.019 & 174.68 \\
\hline \multirow[t]{2}{*}{$\mathrm{J}$} & J & 0.023 & -1.81 & MS & $M$ & 0.017 & -1.94 \\
\hline & $L$ & 0.023 & 176.1 & & $S$ & 0.017 & 177.9 \\
\hline
\end{tabular}

other double-terminal feeders, the phase differences of the measured admittances of the two terminals are both in the range of $-90^{\circ}-90^{\circ}$, and thus Criterion 2 is not satisfied. For the DG feeders, the phase difference of the measured admittances of each terminal is in the range of $-90^{\circ}-90^{\circ}$, and Criterion 1 is not satisfied. As for the load feeders, the amplitudes of the measured admittances are almost unchanged, and Criterion 3 is not satisfied. Thus, the feeder EG is judged to be the faulty line.

\subsection{Fault occurs at F2}

When the fault occurs at F2, the measured admittances of the multi-microgrid are shown in Table 6. In this case, it can be seen from Table 6 that the pre- and post-fault phase difference of the measured admittances of $N$ terminal is $167.32^{\circ}$, and thus Criterion 1 is satisfied. For the double-terminal feeders, the phase differences of the measured admittances of the two terminals are both in the range of $-90^{\circ}-90^{\circ}$, so Criterion 2 is not satisfied. For the other DG feeders, the phase difference of the measured admittances of each terminal is in the range $-90^{\circ}-90^{\circ}$, and Criterion 1 is not satisfied. As for the load feeders, the amplitudes of the measured admittances are almost unchanged, and thus Criterion 3 is not satisfied. Therefore, feeder L7 is judged to be the faulty line.

\subsection{Fault occurs at F3}

When the fault occurs at F3, the measured admittances of the multi-microgrid are shown in Table 7.

In this case, it can be seen from Table 7 that the prefault measured admittance amplitude of $\mathrm{Z}$ terminal is 0.009 . It changes to 6.587 after the fault occurrence, an increase of approximate 730 times. Thus, Criterion 3 is satisfied. As for the other load feeders, the amplitudes of the measured admittances are almost unchanged, so Criterion 3 is not satisfied. The phase differences of the measured admittances of the double-terminal feeders are both in the range of $-90^{\circ}-90^{\circ}$, so Criterion 2 is not satisfied. For the DG feeders, the phase difference of the measured admittances of each terminal is in the range of $-90^{\circ}-90^{\circ}$, and Criterion 1 is not satisfied. Thus, feeder L6 is judged as the faulty line.

From the above simulation results, the fault situations are consistent with the theoretical analysis in Section 3. The fault locations can be determined by the protection criterions in Section 4. Thus, the faulty lines can be removed in time, and the protection function is achieved.

\section{Conclusions}

A new protection scheme for internal faults of multimicrogrid is proposed in this paper, which considers the 
Table 5 Measured admittance results when the F1 fault occurs

\begin{tabular}{|c|c|c|c|c|c|c|c|c|c|}
\hline Feeder & Terminal & $\Delta \varphi_{\mathrm{i}}(\mathrm{O})$ & $\Delta \varphi(\mathrm{o})$ & $\left|G_{1 i}\right|$ & Feeder & Terminal & $\Delta \varphi_{\mathrm{i}}(\mathrm{O})$ & $\Delta \varphi(0)$ & $\left|G_{1 i}\right|$ \\
\hline \multirow[t]{2}{*}{$\overline{\mathrm{EG}}$} & $E$ & -41.50 & -48.64 & 1.467 & L5 & $x$ & 158.67 & -14.25 & 0.004 \\
\hline & G & -22.12 & 150.99 & 0.149 & L6 & Z & -1.54 & 0.22 & 0.009 \\
\hline L1 & C & -1.12 & 0.01 & 0.015 & L7 & N & 171.36 & -3.74 & 0.008 \\
\hline L2 & F & 156.15 & -17.67 & 0.015 & TY & $T$ & -15.32 & -13.39 & 0.006 \\
\hline L3 & $H$ & -1.54 & -0.05 & 0.012 & & Y & 172.58 & -4.34 & 0.006 \\
\hline$\llcorner 4$ & $R$ & -1.58 & 0.18 & 0.012 & L8 & $Q$ & -1.76 & -0.1 & 0.012 \\
\hline \multirow[t]{2}{*}{$\mathrm{KP}$} & K & 140.45 & -29.76 & 0.007 & $A D$ & $A$ & -34.7 & -34.3 & 0.729 \\
\hline & $P$ & -20.16 & -18.13 & 0.007 & & D & 138.88 & -35.8 & 1.479 \\
\hline \multirow[t]{2}{*}{$\mathrm{JL}$} & J & -19.35 & -17.54 & 0.025 & MS & M & -10.58 & -8.64 & 0.018 \\
\hline & L & 160.82 & -15.28 & 0.024 & & S & 169.23 & -8.67 & 0.018 \\
\hline
\end{tabular}

Table 6 Measured admittance results when the F2 fault occurs

\begin{tabular}{|c|c|c|c|c|c|c|c|c|c|}
\hline Feeder & Terminal & $\Delta \varphi_{\mathrm{i}}(\mathrm{O})$ & $\Delta \varphi(\mathrm{o})$ & $\left|G_{1 i}\right|$ & Feeder & Terminal & $\Delta \varphi_{\mathrm{i}}(\mathrm{O})$ & $\Delta \varphi(\mathrm{o})$ & $\left|G_{1 i}\right|$ \\
\hline \multirow[t]{2}{*}{ EG } & $E$ & -27.42 & -34.56 & 0.008 & L5 & $x$ & 166.32 & -6.6 & 0.031 \\
\hline & $G$ & 155.63 & -30.76 & 0.008 & L6 & Z & -1.77 & -0.01 & 0.009 \\
\hline L1 & $C$ & -1.12 & 0.01 & 0.015 & L7 & $N$ & -17.58 & 167.32 & 6.718 \\
\hline L2 & F & 154.84 & -18.98 & 0.015 & TY & $T$ & -68.12 & -66.19 & 0.031 \\
\hline L3 & $H$ & -1.59 & -0.1 & 0.012 & & Y & 109.4 & -67.52 & 0.029 \\
\hline L4 & $R$ & -1.58 & 0.18 & 0.013 & L8 & $Q$ & -1.55 & 0.11 & 0.012 \\
\hline \multirow[t]{2}{*}{$\mathrm{KP}$} & K & 160.4 & -9.81 & 6.28 & $A D$ & $A$ & -17.18 & -16.78 & 0.02 \\
\hline & P & -19.65 & -17.62 & 6.729 & & $D$ & 163.28 & -11.4 & 0.02 \\
\hline \multirow[t]{2}{*}{ JL } & J & -44.69 & -42.88 & 0.958 & MS & M & -55.63 & -53.69 & 0.039 \\
\hline & L & 144.56 & -31.54 & 6.284 & & s & 131.26 & -46.64 & 0.03 \\
\hline
\end{tabular}

Table 7 Measured admittance results when the F3 fault occurs

\begin{tabular}{|c|c|c|c|c|c|c|c|c|c|}
\hline Feeder & Terminal & $\Delta \varphi_{\mathrm{i}}(\mathrm{O})$ & $\Delta \varphi(\mathrm{o})$ & $\left|G_{1 i}\right|$ & Feeder & Terminal & $\Delta \varphi_{\mathrm{i}}(\mathrm{O})$ & $\Delta \varphi(\mathrm{o})$ & $\left|G_{1 i}\right|$ \\
\hline \multirow[t]{2}{*}{$\overline{E G}$} & $E$ & -24.7 & -31.84 & 0.006 & L5 & $x$ & 145.628 & -27.3 & 0.116 \\
\hline & G & 155.43 & -30.39 & 0.006 & L6 & Z & -1.65 & 0.11 & 6.587 \\
\hline L1 & $C$ & -1.31 & -0.18 & 0.015 & L7 & $\mathrm{N}$ & 161.23 & -13.87 & 0.011 \\
\hline L2 & F & 166.83 & -6.99 & 0.015 & TY & $\mathrm{T}$ & -46.36 & -44.43 & 0.703 \\
\hline L3 & $H$ & -1.49 & 0 & 0.012 & & Y & 162.86 & -14.12 & 6.56 \\
\hline L4 & $R$ & -1.41 & 0.35 & 0.012 & L8 & Q & -18.07 & -16.41 & 0.012 \\
\hline \multirow[t]{2}{*}{ KP } & K & 166.34 & -3.87 & 0.009 & $A D$ & A & -8.86 & -8.46 & 0.02 \\
\hline & P & -12.36 & -10.33 & 0.009 & & D & 173.24 & -1.48 & 0.02 \\
\hline \multirow[t]{2}{*}{$J \mathrm{~L}$} & $J$ & -44.35 & -42.54 & 0.278 & MS & M & -44.52 & -42.58 & 0.703 \\
\hline & L & 139.79 & -36.31 & 0.374 & & $S$ & 137.56 & -40.4 & 0.376 \\
\hline
\end{tabular}


control characteristics of the DGs and the interconnection and interaction among adjacent microgrids. The current and voltage characteristics of different feeders are analyzed for faults at different positions of the multimicrogrid. The fault location is realized by comparing the phase differences and amplitudes of measured admittances of the feeders.

The change of the measured bus admittance is the result of the joint action of voltage and current. The change information of the pre- and post-fault measured admittance amplitudes is significant and can be defined as the protection criterion for the load feeders. Furthermore, the phase difference of the measured admittances is defined as another protection criterion for the doubleterminal and DG feeders where it is not a fixed value, but a phase interval. A large redundancy is also introduced in the derivation of the protection criterion and faults at different feeders of the multi-microgrid can be effectively distinguished. Therefore, the fault detection and location can be implemented.

However, for high-impedance faults (HIFs), the change characteristics of current and voltage are not significant and can lead to the failure of Criterion 3. Thus, HIFs at load feeders might not be detected, which is the main limitation of the proposed method.

A multi-microgrid consisted of three $10 \mathrm{kV}$ submicrogrids is established in PSCAD/EMTDC. To examine the effectiveness of the protection scheme, phase-phase-to-ground faults are simulated at four different locations. Theoretical analysis and system simulation results demonstrate the superiority and accuracy of the proposed scheme.

\section{Acknowledgements}

Not applicable.

\section{Authors' contributions}

The paper was a collaborative effort among the authors. FZ performed the simulation, analyzed the data, and wrote the paper. LM provided critical comments and supervised the related research work. All authors read and approved the final manuscript.

\section{Availability of data and materials}

Please contact author for data requests.

\section{Competing interests}

The authors declare that they have no competing interests.

Received: 14 September 2018 Accepted: 21 May 2019

Published online: 28 June 2019

\section{References}

1. Laaksonen, H. J. (2010). Protection principles for future microgrids. IEEE Transactions on Power Electronics, 25(12), 2910-2918.

2. Solanki, A., Nasiri, A., Bhavaraju, V., Familiant, Y. L., \& Fu, Q. (2016). A new framework for microgrid management: Virtual droop control. IEEE Transactions on Smart Grid, 7(2), 554-566.

3. Li, H., Eseye, A. T., Zhang, J., \& Zheng, D. (2017). Optimal energy management for industrial microgrids with high-penetration renewables. Protection \& Control of Modern Power Systems, 2(1), 1-12.

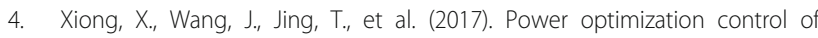
microgrid cluster. Electric Power Automation Equipment, 37(9), 10-17.

5. Liu, N., Zhao, J., Wang, J., et al. (2018). A trading model of PV microgrid cluster based on cooperative game theory. Transactions of China Electrotechnical Society, 33(8), 1903-1910.

6. Min, Z., Ying, C., Chen, S., \& Huang, X. (2015). Characteristic analysis of multi-microgrids and a pilot project design. Power System Technology, 39(6), 1469-1476.

7. Rajaei, N., Ahmed, M. H., \& Salama, M. M. A. (2014). Fault current management using inverter-based distributed generators in smart grids. IEEE Transactions on Smart Grid, 5(5), 2183-2193.

8. Camacho, A., Castilla, M., \& Miret, J. (2015). Active and reactive power strategies with peak current limitation for distributed generation inverters during unbalanced grid faults. IEEE Transactions on Industrial Electronics, 62(3), 1515-1525.

9. Zamani, M. A., Yazdani, A., \& Sidhu, T. S. (2012). A control strategy for enhanced operation of inverter-based microgrids under transient disturbances and network faults. IEEE Transactions on Power Delivery, 27(4), 1737-1747.

10. Lopes, J. A. P., Moreira, C. L., \& Madureira, A. G. (2006). Defining control strategies for microgrids islanded operation. IEEE Transactions on Power Systems, 21(2), 916-924.

11. Laaksonen, H. (2015). Protection scheme for island operated mediumvoltage microgrid. International Review of Electrical Engineering, 10(4), 510-519.

12. Huang, W. T., Tai, N. L., \& Yang, X. (2014). Inverse-time low-impedance protection scheme for microgrids. Proceedings of the CSEE, 34(1), 105-114.

13. Dewadasa, M., Ghosh, A., \& Ledwich, G. (2010). Fold back current control and admittance protection scheme for a distribution network containing distributed generators. IET Generation Transmission \& Distribution, 4(8), 952-962.

14. Dewadasa, M., Majumder, R., Ghosh, A., \& Ledwich, G. (2009). Control and protection of a microgrid with converter interfaced microsources. In 2009 International Conference on Power Systems (ICPS '09) (pp. 1-6).

15. Bukhari, S. B. A., Zaman, M. S. U., Haider, R., Oh, Y.-S., \& Kim, C.-H. (2017). A protection scheme for microgrid with multiple distributed generations using superimposed reactive energy. International Journal of Electrical Power \& Energy Systems, 92, 156-166.

16. Zhang, B., Hao, Z., \& Bo, Z. (2016). New development in relay protection for smart grid. Protection \& Control of Modern Power Systems, 1(1), 1-14.

17. Rocabert, J., Luna, A., \& Blaabjerg, F. (2012). Control of power converters in AC microgrids. IEEE Transactions on Power Electronics, 27(11), 4734-4749.

18. Kroposki, B., Lasseter, R., \& Ise, T. (2008). Making microgrid work. IEEE Power \& Energy Magazine, 6(3), 40-53.

19. Soshinskaya, M., Crijns-Graus, W. H. J., \& Guerrero, J. M. (2014). Microgrids: Experiences, barriers and success factors. Renewable \& Sustainable Energy Reviews, 40, 659-672.

20. Pan, G. Q., Zeng, D. H., \& Wang, G. (2014). Fault analysis on distribution network with inverter interfaced distributed generations based on PQ control strategy. Proceedings of the CSEE, 34(4), 555-561.

\section{Submit your manuscript to a SpringerOpen ${ }^{\circ}$ journal and benefit from:}

- Convenient online submission

- Rigorous peer review

- Open access: articles freely available online

High visibility within the field

Retaining the copyright to your article

Submit your next manuscript at $>$ springeropen.com 